\title{
转子系统盘轴松动故障动力学建模和仿真研究*
}

\author{
李志农 ${ }^{1}$ 刘 杰 $^{1}$ 卢文秀 $^{2}$ 褚福磊 $^{2}$ \\ (1. 南昌航空大学无损检测教育部重点实验室 南昌 330063; \\ 2. 清华大学机械工程系 北京 100084)
}

摘要: 现有的松动故障动力学特性研究主要集中在非转动部件的配合松动上, 而转动部件松动故障却忽略了。针对此不足, 采用 Hertz 接触理论, 建立了盘轴碰摩模型和盘轴松动转子系统的动力学模型, 并进行了仿真研究。讨论了转轴转速、盘轴 弹性模量、盘轴间隙、转轴阻尼、转盘阻尼、转动阻尼、转盘初始转速对转盘运动状态的影响以及转盘振动特性、盘轴位移 差的变化和盘轴运动轨迹。结果表明: 转轴转速、盘轴弹性模量、盘轴间隙、转轴阻尼、转盘阻尼、转动阻尼、转盘初始转 速对转盘运动状态有明显的影响; 在某些转速下, 拍振现象会出现在转轴的波形中, 其原因可能是由盘轴碰摩或者盘轴碰撞 所导致, 在频谱图中会出现二倍频、三倍频等高次谐波成分, 随盘轴转速差的增大, 高倍频的频率也会增多; 当转盘转速趋 于平稳时, 盘轴位移差也会趋于平稳; 锯齿现象会出现在转轴转盘运动轨迹中, 盘轴摩擦力方向的改变会造成锯齿方向不确 定, 且转盘的锯齿现象表现更加明显。研究成果对盘轴松动故障的动力学特性研究具有重要的参考价值。

关键词: 盘轴松动; 非线性振动; Hertz 接触; 故障诊断; 动力学特性

中图分类号: TH113.1；0322

\section{Research on Dynamic Modeling and Simulation of Rotors with Loose Disc}

\author{
LI Zhinong $^{1} \quad$ LIU Jie $^{1} \quad$ LU Wenxiu $^{2} \quad$ CHU Fulei ${ }^{2}$
}

(1. Key Laboratory of Nondestructive Testing, Ministry of Education, Nanchang Hangkong University, Nanchang 330063;

2. Department of Mechanical Engineering, Tsinghua University, Beijing 100084)

\begin{abstract}
The traditional research on the dynamic characteristics of rotor system with loose fault mainly focus on the looseness of the non-rotating parts. However, the loose fault of the rotating parts is neglected. Based on this deficiency, a rotor-disc rubbing model is established by Hertzian impact-contact theory, and the motion equation of rotor system with loose disc is also established. The fourth-order Runge-Kutta method is used to solve this equation. The influence factors of the disc motion state, disc vibration characteristics, variation of displacement between loose disc and shaft, and trajectory of shaft and disc are discussed. The research results show that some factors, such as the shaft speed, elasticity modulus of shaft and disc, clearance between loose disc and shaft, shaft damping, disc damping, rotational damping, and initial speed of loose disc have a major effect on the motion state of loose disc. The waveform of the rotating shaft may have the phenomenon of beat vibration at certain rotating speed, this phenomenon may be caused by the loose disc and shaft's vibration or collision. Some high frequency multiplication occurs in the spectrum, and the multiplication of high frequency increases with the increases of disc and shaft's speed difference. The displacement between loose disc and shaft tends to be stable as the turntable speed tends to be stable. The sawtooth phenomenon occurs in the trajectory diagram of the loose disc and shaft. The change of the frictional force of the disc-shaft leads to the inconsistency of the saw tooth direction, and the sawtooth phenomena of disc is more obvious. The research results have an important reference value for the research of dynamic characteristics of rotor system with loose disc.
\end{abstract}

Key words: rotors with loose disc; nonlinear vibration; hertz contact; fault diagnosis; dynamic characteristic

\footnotetext{
* 国家自然科学基金(51675258, 51875301, 51075372)、机械系统与振动国家 重点实验室课题(MSV201914)和机械传动国家重点实验室开放基金 (SKLMT-KFKT-201514)资助项目。20190503 收到初稿, 20190909 收到修 改稿
} 


\section{0 前言}

松动故障是旋转机械常见的故障之一, 它是装 配质量不高和长期振动导致机械产生的。目前, 关 于松动故障的研究已经取得很大的进展 ${ }^{[1-8]}$ 。文献[9] 研究了具有基础松动的双线性模型的不平衡响应及 模型混沌分岔行为; 文献[10-13]从理论和实验研究 了在不同转速下, 非线性振动系统出现的不同复杂 的动力学现象。文献[14-15]通过数值仿真方法分析 了带有一端及两端支座松动故障的转子-轴承系统 的复杂运动现象。文献[16]针对恒转速下轴承-转子 系统的基座松动问题，提出了基于非线性度量的评 估方法, 同时模型中还考虑了分段线性刚度, 阻尼 力和非线性弹性力等影响因素。然而, 现有的松动 故障研究对象主要集中在非旋转部件上, 如基座松 动, 轴承座底座松动等, 而对旋转部件的松动故障 研究却被忽略了。转盘作为转子系统的主要做功部 件, 它的工作环境复杂且恶劣, 在此情况下, 盘轴 大多采用过盈配合的装配方式, 这种配合方式很容 易产生盘轴装配界面的松动, 从而导致盘轴连接的 非线性, 并导致不平衡质量位置不确定性。这种转 动部件松动问题应引起高度重视, 否则, 将会在工 程实际中带来很大的安全隐患, 给国民经济造成巨 大损失。目前, 文献[17-18]考虑了大间隙盘轴松动 模型, 分析在不同转盘转速情况下, 转子系统的动 力学特性变化, 其中假设转盘以恒定转速工作, 同 时盘轴总处于接触状态。然而, 这种建模明显与客 观实际不符合。因为在实际工程中, 当盘轴存在间 隙时, 盘轴之间会发生碰摩运动, 转盘转速并不一 定是恒定的，盘轴之间的碰撞可能导致盘轴并不 是一直处于接触状态。因此需要进一步研究在间 隙配合情况下盘轴松动故障动力学模型建立的问 题。基于 Hertz 接触理论, 本文先建立了盘轴接触 模型, 接着基于所建立的模型建立系统的动力学 微分方程, 并进行数值仿真, 分别讨论各个影响 因素对转盘运动状态的影响、转盘的振动特性、 盘轴位移差和盘轴运动轨迹, 为盘轴松动故障动 力学特性研究提供一种新方法。

\section{1 数学模型}

\section{1 基于 Hertz 接触理论的碰摩模型}

盘轴系统碰摩模型如图 1 所示, 在图 1 中, 各 符号含义如下: $o_{1}$ 为转轴的形心, 坐标为 $\left(x_{1}, y_{1}\right)$; $\mathrm{O}_{2}$ 为转盘的形心, 坐标为 $\left(x_{2}, y_{2}\right) ; c$ 为转盘的质心; $\theta$ 为转盘的转动角位移; $F_{r}$ 为转盘对转轴径向力; $F_{t}$ 为转盘对转轴切向摩擦力。

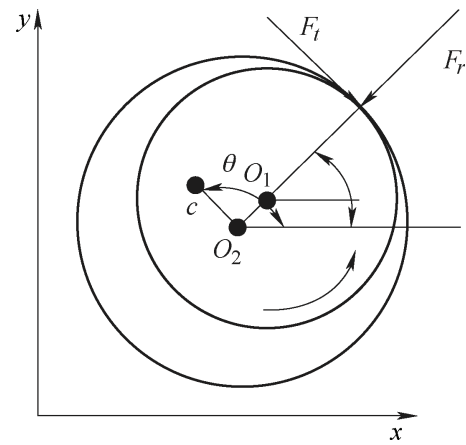

图 1 盘轴系统碰摩模型

为了研究方便, 这里假设如下 ${ }^{[19]}$ 。

(1) 在整个过程中, 转盘转轴是刚性体; 在两 者接触是产生的碰撞力 (也称径向力 $F_{r}$ ) 出现在转 盘转轴的公法线上。

(2) 在挤压接触区域, 转盘转轴会出现的局部弹 性变形, 同时, 在此过程中, 忽略两者的阻尼效应。

(3) 盘轴接触点处的切向摩擦力为 $F_{t}=\mu F_{r}$, 其 中, $\mu$ 为摩擦因数。

为简化分析, 假设转盘为具有一定厚度和宽度 的圆盘, 转轴为具有一定宽度和厚度的圆孔, 这样, 转盘转轴的碰撞问题可看作为两个圆的内接触问 题。根据 Hertz 接触定律, 转盘转轴碰撞引起的法 向碰撞力为

$$
F_{r}=\left(\frac{\delta}{\alpha}\right)^{\frac{3}{2}}
$$

式中 $\delta$ 一径向嵌入深度;

$\alpha$ 一结构常量, 其表达式可以表示为 ${ }^{[20]}$

$$
\alpha=0.8 \times \sqrt[3]{\frac{9}{16}\left(\frac{1-v_{1}^{2}}{E_{1}}+\frac{1-v_{2}^{2}}{E_{2}}\right)^{2}\left(\frac{1}{R_{1}}-\frac{1}{R_{2}}\right)}
$$

式中 $v_{i}(i=1,2)$ 一一转轴和转盘的泊松比;

$E_{i}(i=1,2)$ 一一转轴和转盘的弹性模量;

$R_{i}(i=1,2)$ 一一转盘和转轴的撞击局部表面 曲率半径。

由于转盘和转轴的碰撞局部表面曲率半径相差 很小, 即: $R_{1} \approx R_{2}$, 盘轴半径间隙 $h \ll R_{1}$, 则 $\alpha$ 可 表达为

$$
\alpha=0.8 \times \sqrt[3]{\frac{9}{16}\left(\frac{1-v_{1}^{2}}{E_{1}}+\frac{1-v_{2}^{2}}{E_{2}}\right)^{2}\left(\frac{h}{R_{1}^{2}}\right)}
$$

转盘转轴的径向作用力 $F_{r}$ 可表达为

$$
F_{r}=S(\delta)\left(\frac{\delta}{\alpha}\right)^{\frac{3}{2}}
$$


此时, $\delta=r-h, r$ 为转子径向位移; 阶跃函数

$$
S(\delta)= \begin{cases}1 & \delta \geqslant 0 \\ 0 & \delta<0\end{cases}
$$

转轴在转盘转轴接触点的切向速度为

$$
V_{t 1}=\omega R_{1}+\dot{y}_{1}\left(\frac{x_{1}-x_{2}}{r}\right)-\dot{x}_{1}\left(\frac{y_{1}-y_{2}}{r}\right)
$$

转盘在转盘转轴接触点的切向速度为

$$
V_{t 2}=\dot{\theta} R_{2}+\dot{y}_{2}\left(\frac{x_{1}-x_{2}}{r}\right)-\dot{x}_{2}\left(\frac{y_{1}-y_{2}}{r}\right)
$$

令 $\Delta V=V_{t 1}-V_{t 2}$, 则 $F_{t}$ 可表示为

$$
F_{t}=\operatorname{sgn}(\Delta V) \mu F_{r}
$$

其中, $\operatorname{sgn}(\Delta V)$ 为符号函数, 其表达式为

$$
\operatorname{sgn}(\Delta V)=\left\{\begin{array}{cc}
1 & \Delta V>0 \\
0 & \Delta V=0 \\
-1 & \Delta V<0
\end{array}\right.
$$

由此可得到转盘对转轴的作用力在两坐标轴上的分 量为

$$
\left\{\begin{array}{l}
F_{x} \\
F_{y}
\end{array}\right\}=-\frac{1}{r}\left[\begin{array}{cc}
F_{r} & -F_{t} \\
-F_{t} & F_{r}
\end{array}\right]\left\{\begin{array}{l}
x_{1}-x_{2} \\
y_{1}-y_{2}
\end{array}\right\}
$$

\section{2 盘轴松动转子系统运动方程}

盘轴松动转子系统模型如图 2 所示, 系统模型 由转子和松动转盘两部分组成, 其中, 转子是具有 集中质量和刚性支承性质。文献[17-18]发现陀螺效 应对盘轴松动转子系统动力学特性影响较小, 为简 化研究过程, 忽略陀螺效应对模型系统的影响。应 用数值分析方法对动力学微分方程进行分析, 得到 盘轴松动转子系统的动力学特性。

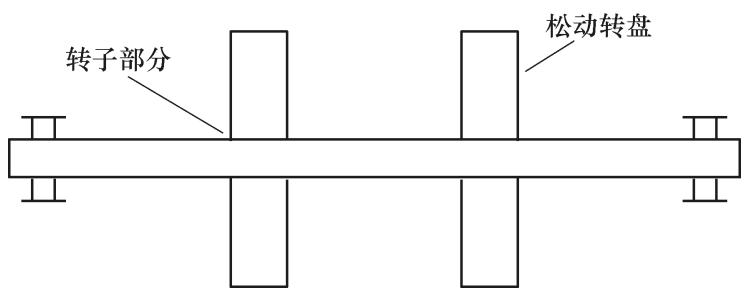

图 2 盘轴松动转子系统模型图

对于转子部分, 转轴的振动方程为

$$
\left\{\begin{array}{l}
m_{1} \ddot{x}_{1}+c_{1} \dot{x}_{1}+k x_{1}=m_{1} e_{1} \omega^{2} \cos (\omega t)+F \\
m_{1} \ddot{y}_{1}+c_{1} \dot{y}_{1}+k y_{1}=m_{1} e_{1} \omega^{2} \sin (\omega t)-m_{1} g+F_{y}
\end{array}\right.
$$

式中 $m_{1}$ 一转轴的质量;

$c_{1}$ ——转轴的阻尼系数;

$e_{1}$ —转轴的偏心距离;

$\omega$ 一一转轴的自转速度;

$g$ 一一模型系统的重力加速度。

对于转盘部分, 转盘的振动方程为

$$
\left\{\begin{array}{l}
m_{2} \ddot{x}_{2}+c_{2} \dot{x}_{2}=m_{2} e_{2} \dot{\theta}^{2} \cos \theta+m_{2} e_{2} \ddot{\theta} \sin \theta-F \\
m_{2} \ddot{y}_{2}+c_{2} \dot{y}_{2}=m_{2} e_{2} \dot{\theta}^{2} \sin \theta-m_{2} e_{2} \ddot{\theta} \cos \theta-m_{2} g-F_{y}
\end{array}\right.
$$

式中 $m_{2}$ 一转盘的质量;

$c_{2}$ 一转盘的阻尼系数;

$e_{2}$ 一转盘的偏心距离。

转盘的转动振动方程为

$$
J \ddot{\theta}+c_{3} \dot{\theta}=F_{t} R_{2}-m_{2} g e_{2} \cos \theta
$$

式中 $J$ 一转盘的转动惯量;

$c_{3}$ 一转盘的转动阻尼系数。

对式(9) (11)进行量纲一化得到的运动方程为

$$
\left\{\begin{array}{l}
X_{1}^{\prime \prime}=E_{1} \cos \tau+\frac{f_{x}}{\Omega^{2}}-\frac{\xi_{1}}{\Omega} X_{1}^{\prime}-\frac{\eta}{\Omega^{2}} X_{1} \\
Y_{1}^{\prime \prime}=E_{1} \sin \tau-\frac{G}{\Omega^{2}}+\frac{f_{y}}{\Omega^{2}}-\frac{\xi_{1}}{\Omega} Y_{1}^{\prime}-\frac{\eta}{\Omega^{2}} Y \\
X_{2}^{\prime \prime}=E_{2} \theta^{\prime 2} \cos \theta+E_{2} \theta^{\prime \prime} \sin \theta-\frac{f_{x}}{s \Omega^{2}}-\frac{\xi_{2}}{\Omega} X^{\prime} \\
Y_{2}^{\prime \prime}=E_{2} \theta^{\prime 2} \sin \theta-E_{2} \theta^{\prime \prime} \cos \theta-\frac{f_{y}}{s \Omega^{2}}-\frac{\xi_{2}}{\Omega} Y_{2}^{\prime}-\frac{G}{\Omega^{2}} \\
\theta^{\prime \prime}=\frac{M_{1}}{\Omega^{2}}-\frac{M_{2}}{\Omega^{2}} \cos \theta-\frac{\xi_{3}}{\Omega} \theta^{\prime}
\end{array}\right.
$$

式中, $\tau=\omega t, X_{i}=\frac{x_{i}}{h}, Y_{i}=\frac{y_{i}}{h}, X_{i}^{\prime}=\frac{\mathrm{d} x_{i}}{\mathrm{~d} \tau}, Y_{i}^{\prime}=\frac{\mathrm{d} y_{i}}{\mathrm{~d} \tau}$, $X_{i}^{\prime \prime}=\frac{\mathrm{d} X_{i}^{\prime}}{\mathrm{d} \tau}, Y_{i}^{\prime \prime}=\frac{\mathrm{d} Y_{i}^{\prime}}{\mathrm{d} \tau}, \omega_{0}=\sqrt{\frac{k}{m_{1}}}, \Omega=\frac{\omega}{\omega_{0}}, E_{1}=\frac{e_{1}}{h}$, $E_{2}=\frac{e_{2}}{h}, \quad \xi_{1}=\frac{c_{1}}{m_{1} \omega_{0}}, \quad \xi_{2}=\frac{c_{2}}{m_{2} \omega_{0}}, \quad \eta=\frac{k_{1}}{m_{1} \omega_{0}^{2}}$, $G=\frac{g}{\omega_{0}{ }^{2} h}, f_{x}=\frac{F_{x}}{m_{1} \omega_{0}{ }^{2} h}, f_{y}=\frac{F_{y}}{m_{1} \omega_{0}{ }^{2} h}, M_{1}=\frac{F_{t} R_{2}}{J \omega_{0}{ }^{2}}$, $M_{2}=\frac{m_{2} g e_{2}}{J \omega_{0}{ }^{2}}, \quad \xi_{3}=\frac{c_{3}}{J \omega_{0}}, \quad s=\frac{m_{2}}{m_{1}} 。$

这里, 采用四阶龙格库塔法对方程组式(12)进 行数值计算。为提高解的精确性和收玫性, 这里采 用的步长为 $\pi / 640$ 。

\section{2 数值分析}

在本模型中, 设定系统参数为 $E_{1}=20, \xi_{1}=$ $0.0029, \eta=1, G=2.94, E_{2}=20, s=1 / 3$, $\xi_{2}=0.0087, \xi_{3}=3 \times 10^{-5}, M_{2}=1.176 \times 10^{-5}, M_{1}$ 代 表盘轴之间的摩擦力矩的量纲一值, 在系统运行中 随盘轴径向嵌入深度, 盘轴相对转速的影响。系统 运动方程无量纲化处理后, $M_{1}$ 被约去, 未出现在系 统方程中。在初始条件为转盘静止条件下, 讨论各 个因素对转盘运动状态的影响、盘轴位移差的变化、 转盘振动特性和盘轴运动轨迹, 分析系统的振动特 性和动力学特性。 


\section{1 转盘运动状态影响因素分析}

在所建立的模型系统中, 分别分析转轴转速 $\Omega$ 、盘轴弹性模量 $E_{1} 、 E_{2} 、 h$ 、转轴阻尼 $\xi_{1}$ 、转 盘阻尼 $\xi_{2}$ 、转动阻尼 $\xi_{3} 、 \theta^{\prime}$ 等物理量对转盘运动状 态的影响, 分析结果对进一步了解盘轴松动模型和 理解故障特征机理具有重要意义。

(1) 转轴转速的影响。

转盘量纲一转速 $\theta^{\prime}$ 随转轴转速 $\Omega$ 的变化规律 如图 3 所示。由图 3 可知, 当 $0.52 \leqslant \Omega \leqslant 0.64$ 时, $\theta^{\prime}$ 值在 0.5 处接近稳定。其中, 图 $3 \mathrm{a}$ 表给出了 $\Omega=0.624$ 的转盘转速变化曲线图。当 $0.64<\Omega \leqslant 0.78$ 时, 转 盘最终进入稳定运转状态, 此时, $\theta^{\prime}$ 在接近 0.50 处 存在小波动, 图 $3 \mathrm{~b}$ 为 $\Omega=0.658$ 时, 转盘转速变化 曲线图。当 $0.78<\Omega \leqslant 0.953$ 时, 转盘运动状态最终 进入稳定运转状态, 此时, $\theta^{\prime}$ 稳定在 $0.44 \sim 0.50$ 中 的某一固定值处, 图 3c 为 $\Omega=0.849$ 时, 转盘转速 变化曲线图。当 $0.953<\Omega \leqslant 1.057$ 时, 转盘运动状 态进入稳定状态, 此时, $\theta^{\prime}$ 在接近 0.5 处存在小波 动, 图 $3 \mathrm{~d}$ 为 $\Omega=1.005$ 时, 转盘转速变化曲线图。 当 $1.057<\Omega \leqslant 1.628$ 时, 转盘运动状态进入稳定转动 状态时, $\theta^{\prime}$ 稳定在 $0.46 \sim 0.50$ 中的某一固定值处, 图 3e 为 $\Omega=1.386$ 时, 转盘转速变化曲线图。当 $1.628<\Omega \leqslant 1.715$ 时, 转盘运动状态不存在稳定情 况, 图 3f 为 $\Omega=1.645$ 时, 转盘转速变化曲线图。

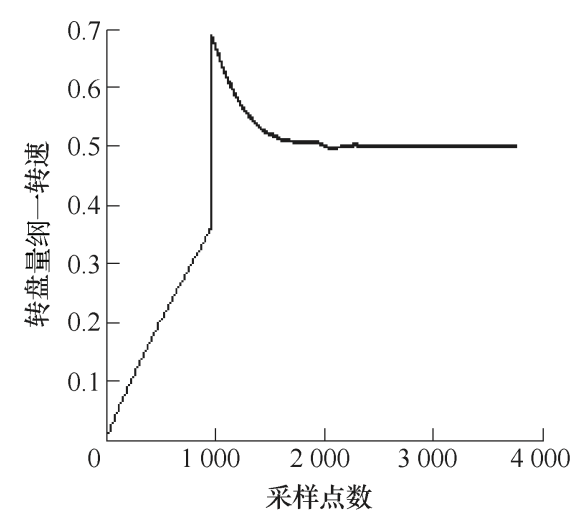

(a) $\Omega=0.624$

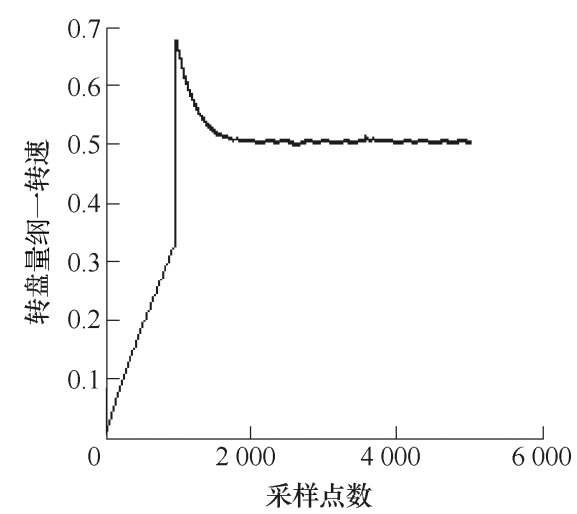

(b) $\Omega=0.658$

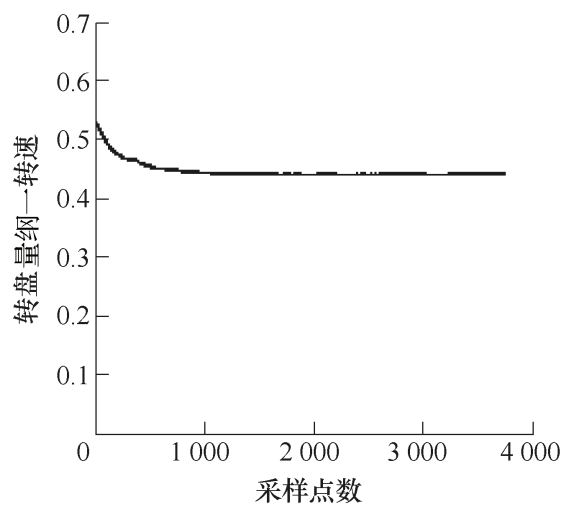

(c) $\Omega=0.849$

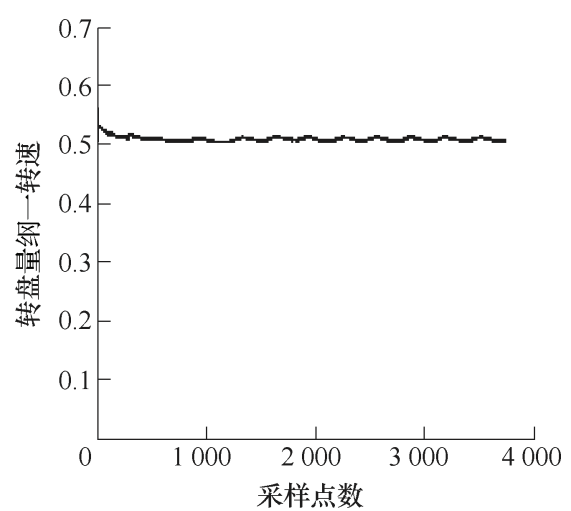

(d) $\Omega=1.005$

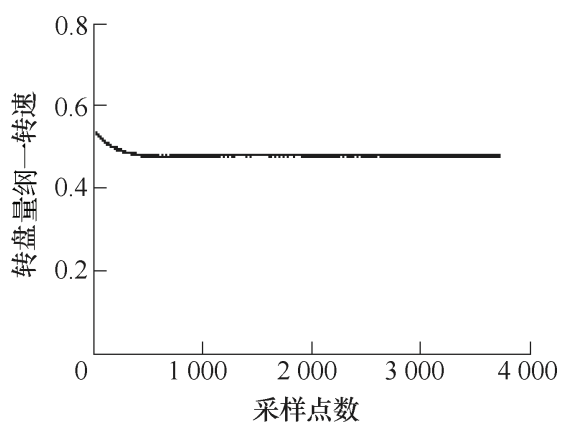

(e) $\Omega=1.386$

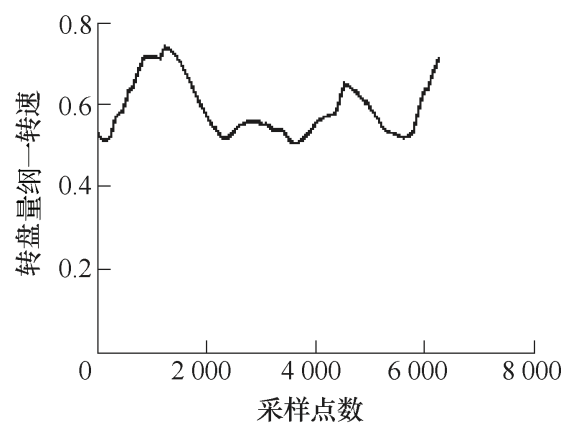

(f) $\Omega=1.645$

图 3 不同转轴转速下, 转盘转速变化曲线图

（2）弹性模量的影响。

由于盘轴接触刚度与盘轴弹性模量呈现线性关 系, 假设转盘转轴材料相同, 则两者的弹性模量 $E_{1}=E_{2}$ 。图 4 为在不同弹性模量情况下, 转盘转速 
变化曲线图。由图 4 可知: 转盘的稳定转速随着弹 性模量的增加而减小, 而转盘运动状态随弹性模量 增加越来越稳定。

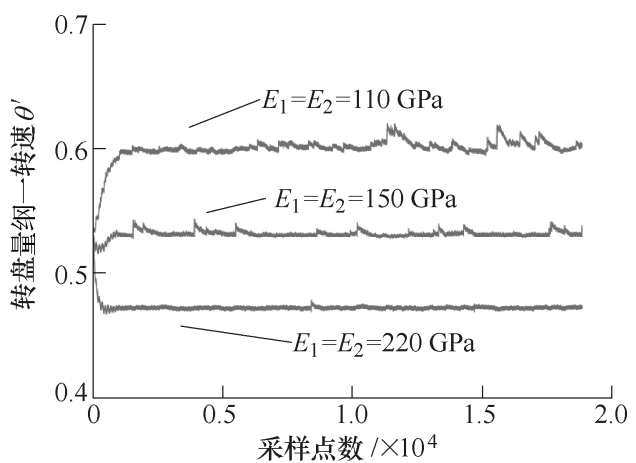

图 4 不同弹性模量, 转盘转速变化曲线图

(3) 盘轴间隙 $h$ 的影响。

研究发现, 当盘轴间隙 $h$ 小于 $3.4 \times 10^{-5} \mathrm{~m}$ 时, 随着间隙增大, 转盘稳态转速也增大, 图 5 给出了 转盘的稳态转速随着盘轴间隙的变化图。当盘轴间 隙大于 $3.4 \times 10^{-5} \mathrm{~m}$ 时, 转盘的稳态转速与转盘量纲 一转速呈现线性关系, 当盘轴间隙逐渐增大时, 转 盘量纲一转速的波动也越来越大。图 6 给出了转盘 转速随着盘轴间隙变化的波动图。

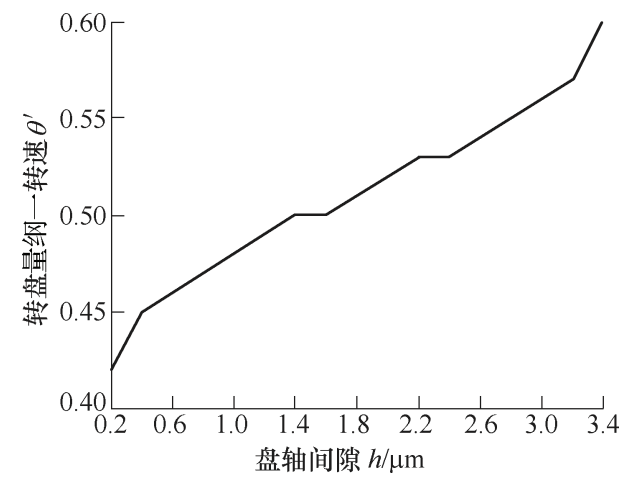

图 5 不同间隙下, 转盘稳态转速变化图

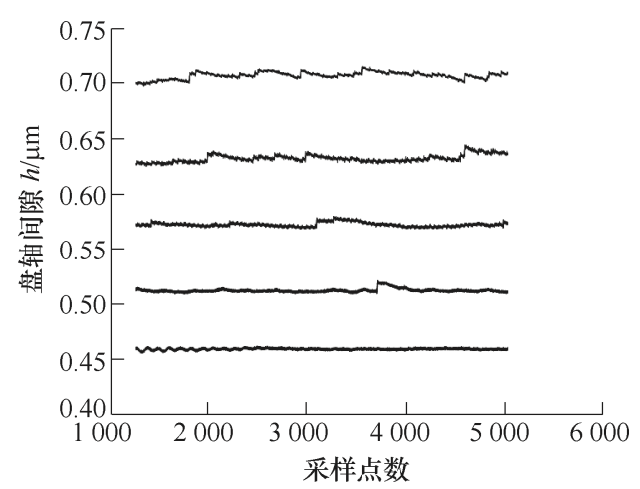

图 6 不同间隙，转盘转速波动图

(4) 阻尼的影响。

先讨论转轴阻尼 $\xi_{1}$ 对转盘量纲一转速的影响。 当 $0 \leqslant \xi_{1} \leqslant 0.024$ 时, 当 $\xi_{1}$ 增大时, $\theta^{\prime}$ 也增大, 且 $\theta^{\prime}$
值会出现小范围波动, 图 7 为转盘转速 $\theta^{\prime}$ 随 $\xi_{1}$ 的变 化图。

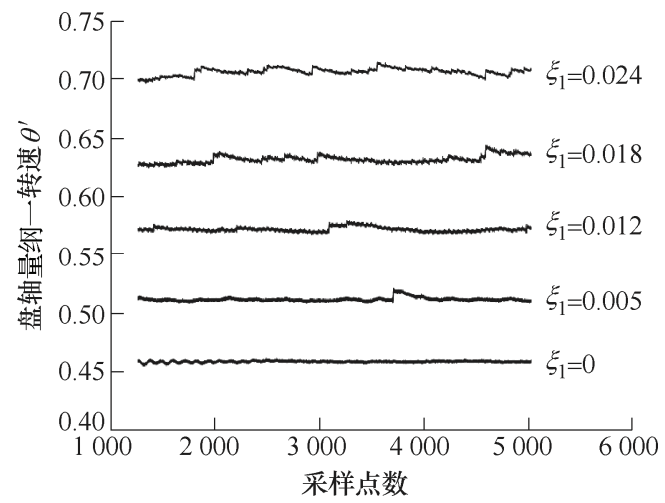

图 7 不同 $\xi_{1}$, 转盘转速 $\theta^{\prime}$ 变化图

在 $0.024<\xi_{1}$ 前提下, 随着 $\xi_{1}$ 的不断增大, 转盘 量纲一转速 $\theta^{\prime}$ 逐渐接近转轴转速, 最终趋近于稳定 值。图 8 为 $\xi_{1}=0.03$ 时, 转盘转速 $\theta^{\prime}$ 变化图。

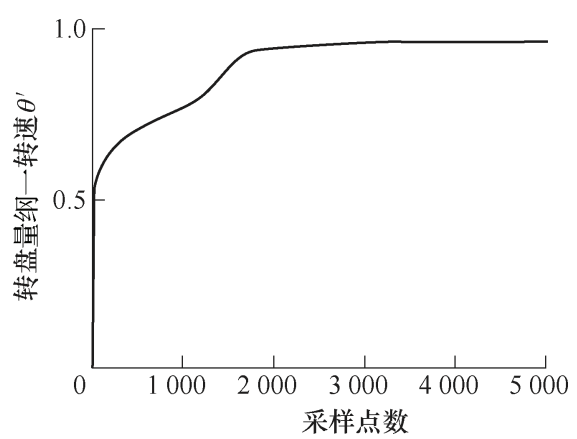

图 $8 \xi_{1}=0.03$, 转盘量纲一转速 $\theta^{\prime}$ 变化图

再讨论转盘阻尼 $\xi_{2}$ 对转盘量纲一转速的影响。 当 $0 \leqslant \xi_{2} \leqslant 0.0095$ 时, 转盘量纲一转速 $\theta^{\prime}$ 与 $\xi_{2}$ 呈现 线性递增关系, 图 9 为量纲一转速 $\theta^{\prime}$ 随 $\xi_{2}$ 的变化图; 当 $0.0095<\xi_{2} \leqslant 0.021$ 时, 当 $\xi_{2}$ 不断地增大时, 转盘 量纲一转速 $\theta^{\prime}$ 也逐渐增大, 且 $\theta^{\prime}$ 出现波动现象, 图 10 为不同 $\xi_{2}$ 情况下, 转盘量纲一转速 $\theta^{\prime}$ 变化图; 在 $0.021<\xi_{2} \leqslant 0.07$ 前提下, 转盘转速 $\theta^{\prime}$ 随着 $\xi_{2}$ 的不 断增加逐渐接近转轴量纲一转速并逐渐趋于稳定, 图 11 为 $\xi_{2}=0.03$ 时, 转盘量纲一转速 $\theta^{\prime}$ 变化图; 当 $0.07<\xi_{2}$ 时, 转盘量纲一转速 $\theta^{\prime}$ 与转轴转速相同, 图 12 为 $\xi_{2}=0.08$ 时, 转盘量纲一转速 $\theta^{\prime}$ 的变化图。

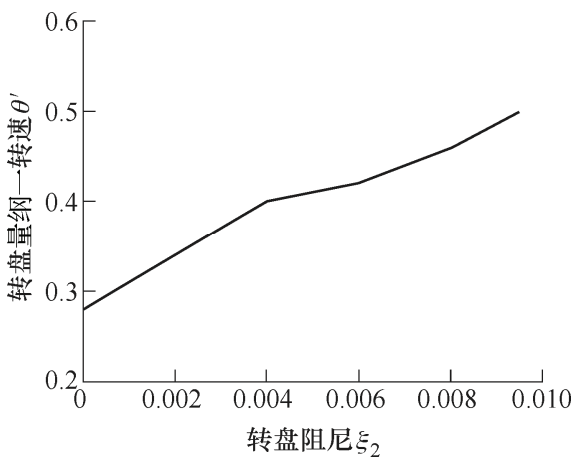

图 9 量纲一转速 $\theta^{\prime}$ 随 $\xi_{2}$ 变化图 


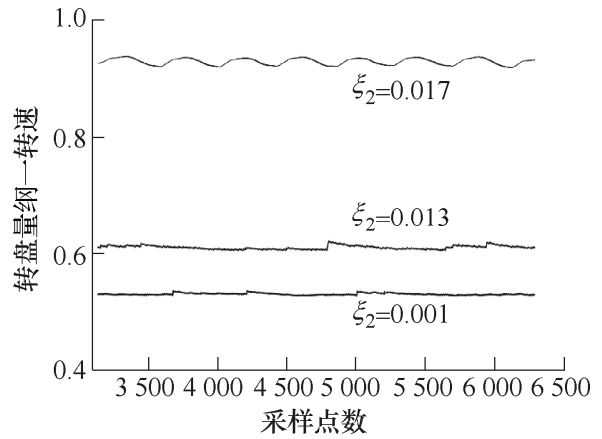

图 10 不同 $\xi_{2}$, 转盘转速 $\theta$ 变化图

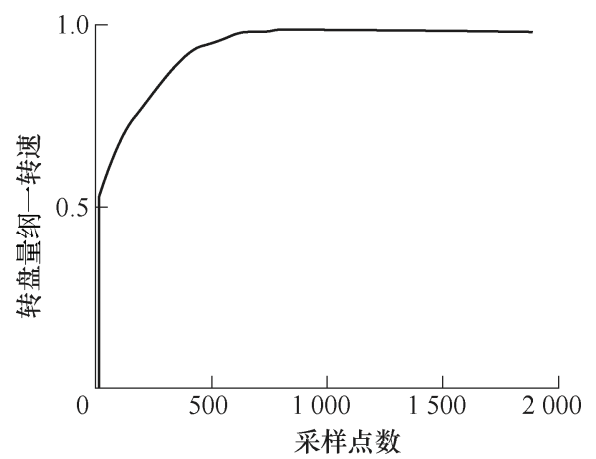

图 $11 \xi_{2}=0.08$, 转盘量纲一转速 $\theta^{\prime}$ 变化图

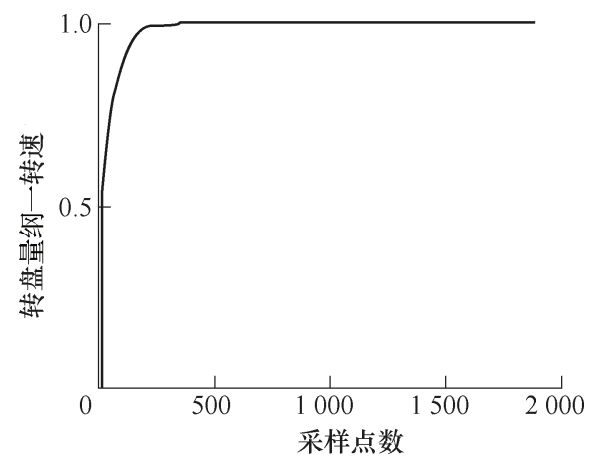

图 12 转盘量纲一转速 $\theta^{\prime}$ 随 $\xi_{2}$ 变化图

最后, 讨论转动阻尼 $\xi_{3}$ 对转盘量纲一转速的影 响, 由研究结果可知: 在 $\xi_{3} \leqslant 34$ 前提条件下, 随着 转动阻尼 $\xi_{3}$ 的增大, 转盘量纲一转速逐渐减小并最 终在某一固定值上下小范围波动, 图 13 给出了转盘 量纲一转速 $\theta^{\prime}$ 随 $\xi_{3}$ 变化图; 由图 14 可知, 在 $34<\xi_{3}$ 前提条件下, 随着时间的不断增加, 转盘量纲一转 速 $\theta^{\prime}$ 会先持续下降情况接着会出现在某一值处小 范围波动现象, 但是, $\theta^{\prime}$ 无法稳定在某一值处; 图 14 为 $\xi_{3}=77$ 时, 转盘量纲一转速 $\theta^{\prime}$ 变化图。

(5) 盘转盘初始转速 $\theta_{0}^{\prime}$ 的影响。

由仿真结果可知: 当 $0 \leqslant \theta_{0}^{\prime} \leqslant 0.2$ 时, 转盘量纲 一转速 $\theta^{\prime}$ 值稳定为 0.47 ; 当 $0.2<\theta_{0}^{\prime} \leqslant 0.7$ 时, 转盘 量纲一转速 $\theta^{\prime}$ 值会在 $0.9 \sim 0.95$ 之间的某一值处出 现波动现象, 但是无法稳定在某一值处; 当 $0.7<\theta_{0}^{\prime} \leqslant 1.5$ 时, 转盘量纲一转速 $\theta^{\prime}$ 值稳处于 1 。

图 15 为在不同初始转速 $\theta_{0}^{\prime}$ 值情况下, 转盘量 纲一转速 $\theta^{\prime}$ 变化曲线图。

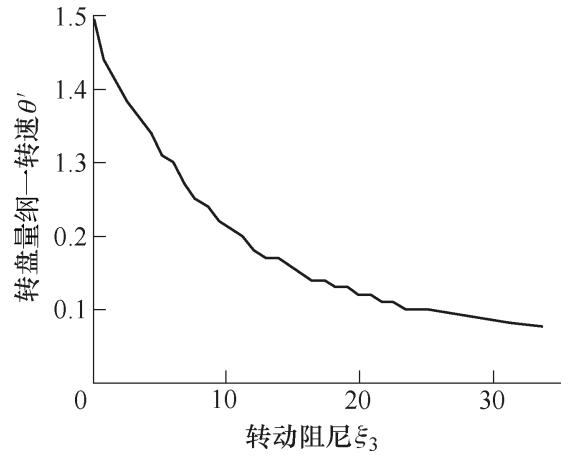

图 13 转盘量纲一转速 $\theta^{\prime}$ 随 $\xi_{3}$ 变化图

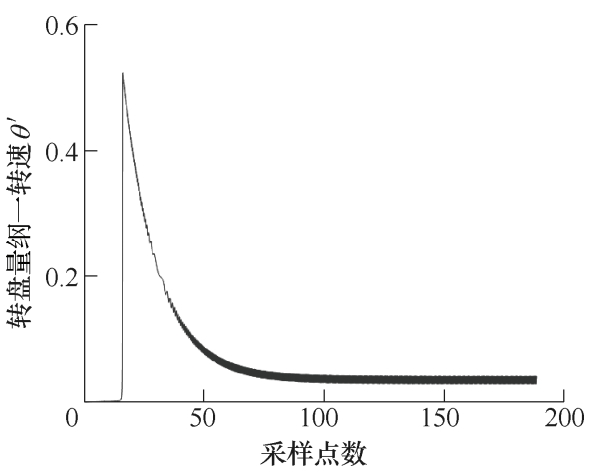

图 $14 \xi_{3}=77$, 转盘量纲一转速 $\theta^{\prime}$ 变化图

由上述结论可知: 当盘轴出现松动故障时, 转 盘运动状态会受到 $\Omega 、 E_{1} 、 E_{2} 、 h 、 \xi_{1} 、 \xi_{2} 、 \xi_{3}$ 、 $\theta_{0}^{\prime}$ 等因素的影响。

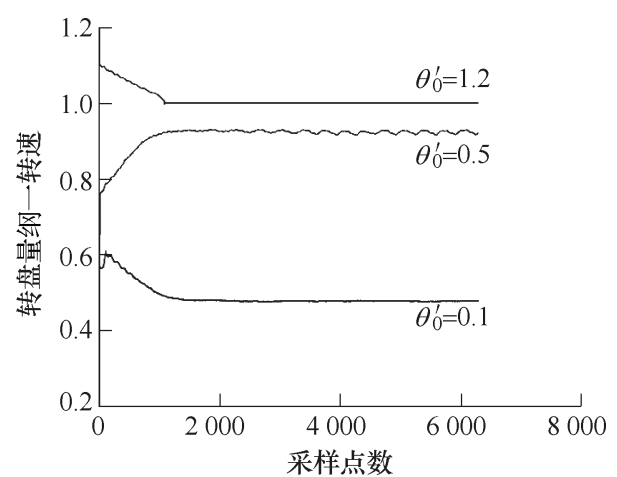

图 15 不同 $\theta_{0}^{\prime}$ 值, 转盘量纲一转速 $\theta^{\prime}$ 变化曲线

\section{2 不同转盘转速, 振动波形图及频率特性分析}

图 16 为转盘与转轴以同一转速运行时, 转轴 $x$ 方向 10 个周期的波形图和频谱图。由频谱图(图 16b) 可得: 频谱成分非常丰富, 既有单倍频, 也存在较多 高倍频, 倍频数越大, 其量纲一幅值越小。当转盘量 纲一转速 $\theta^{\prime}=0.93$ 时, 转轴 $x$ 方向 50 个周期的波形图 和频谱图如图 17 所示。图 17b 的频谱图与传统频谱 图存在很大的区别, 其波形图中出现拍振现象; 拍振 现象的出现是由于转盘与转轴的振动导致的; 其频谱 图中既包含 1 倍频和接近 1 倍频的成分, 也包含高倍 频成分; 一倍频为转轴转动频率, 低倍频为转盘转动 频率, 高倍频为转盘与转轴的碰撞频率。 


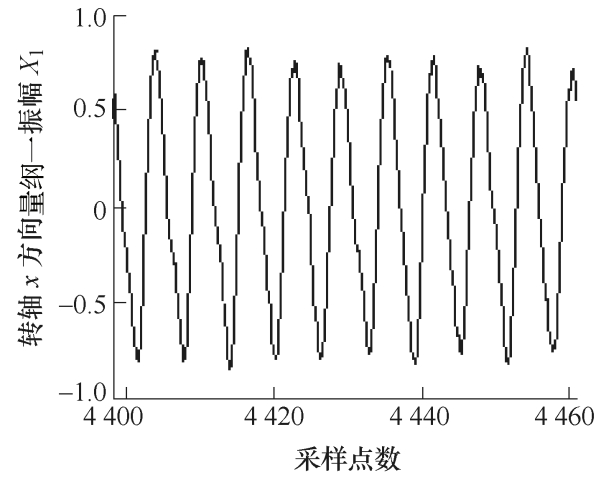

(a) 波形图

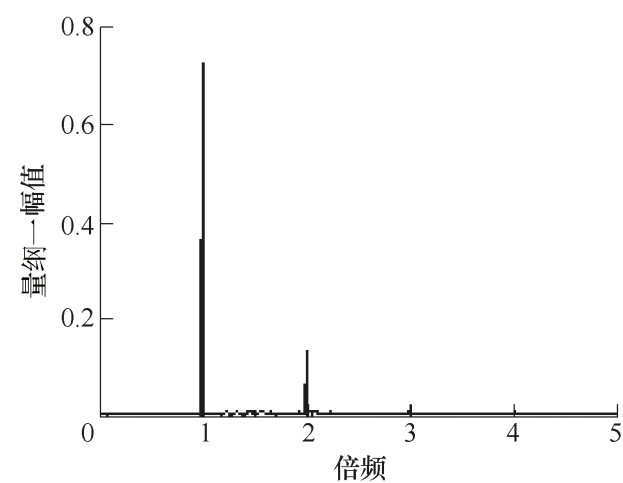

(b) 频谱图

图 16 当转盘转轴同速时, 转轴 $x$ 方向 10 个周期的 波形图、频谱图

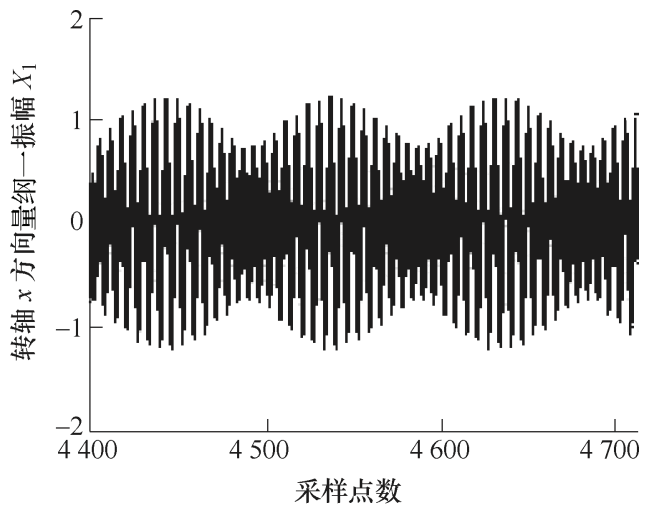

(a) 波形图

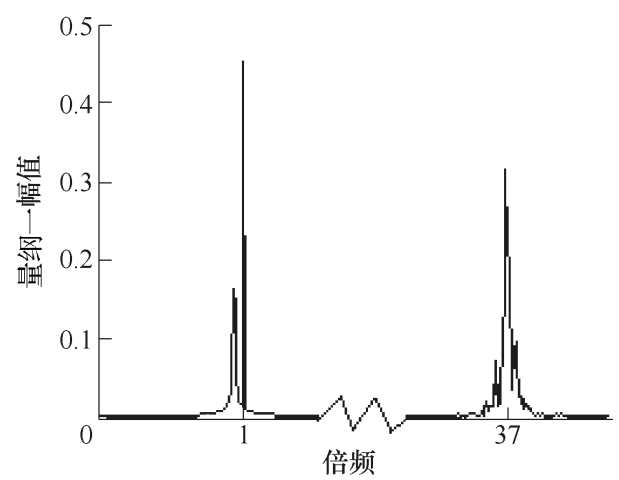

(b) 频谱图

图 $17 \theta^{\prime}=0.93$ 时, 转轴 $x$ 方向 50 个周期的 波形图、频谱图
图 18 为转盘量纲一转速 $\theta^{\prime}=0.45$ 时, 转轴 $x$ 方 向 1 个周期的波形图、频谱图。其波形图中也出现 了拍振现象; 其频谱图中包含一倍频、较少的低倍 频和高倍频; 由于低倍频和高倍频不接近, 显然拍 振现象应当由相接近的转盘与转轴碰撞造成的。

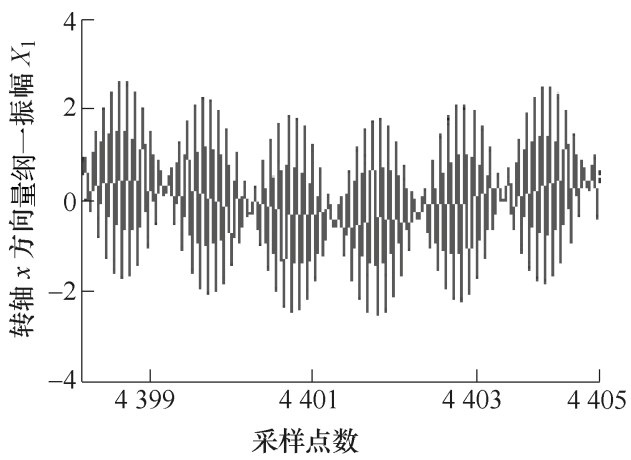

(a) 波形图

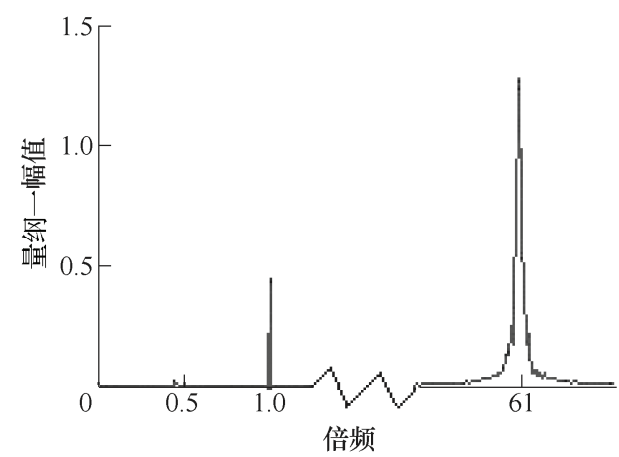

(b) 频谱图

图 $18 \theta^{\prime}=0.45$ 时, 转轴 $x$ 方向 1 个周期的的 波形图、频谱图

由图 16 18 的分析结果可以得出: 随着转盘与 转轴转速差的增加, 高倍频的频率值也在不断地增 加, 这说明转盘与转轴之间的碰摩越来越严重。

综上所述, 在一些的转盘转速下, 转轴的波形 图中会出现拍振现象。这种拍振现象可能是由盘轴 振动导致或盘轴碰撞导致的; 随着盘轴转速差的增 大，频谱图中的高倍频的频率值也在不断增大。

\section{3 盘轴位移差分析}

图 19 为转盘转速变化图及其对应的盘轴位移 差变化图, 由图 19 可知, 当转盘转速处于稳定状态 时，盘轴位移差也处于稳定状态。

\section{4 运动轨迹分析}

图 20 为轴心、盘心运动轨迹图 (转盘量纲一转 速 $\theta^{\prime}=0.5$ ), 表 1 给出了转盘量纲一转速 $\theta^{\prime}=0.5$ 时 运动方程部分稳态数值结果。由轨迹图可知, 锯齿 现象明显出现, 这是由于盘轴间存在碰撞力造成的, 同时锯齿的方向不定的。由表 1 可得： $\Delta V$ 的符号 时正时负, 这是由于当盘轴接触点的切向速度大于 转轴速度时, 摩擦力做负功, 则其符号正好为负; 
当盘轴接触点的切向速度小于转轴速度时, 摩擦力 做正功, 其符号为正; 盘心轨迹图中的锯齿现象较 轴心轨迹图中的锯齿现象更明显, 这是由于转盘的 质量较转轴小, 其惯性也小, 当转盘受到碰摩力时, 其运动状态更容易发生改变。

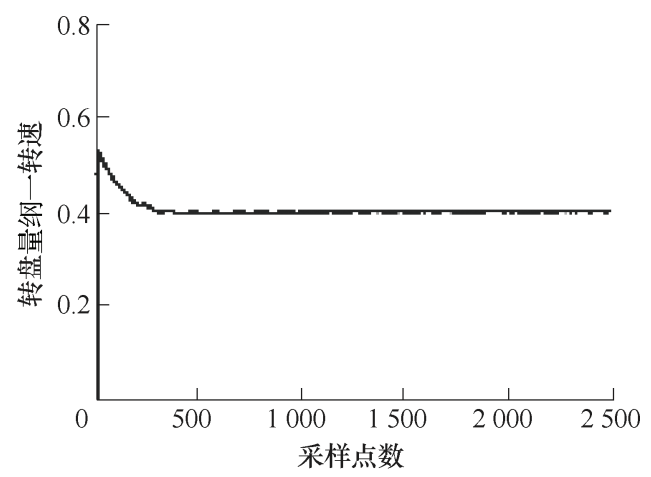

(a) 转盘转速变化图

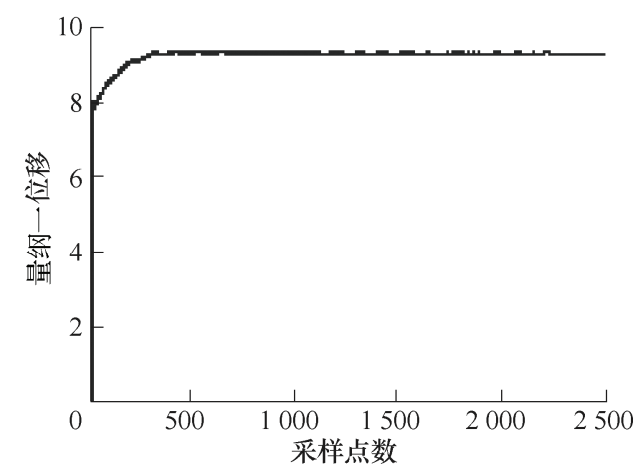

(b) 盘轴位移差变化图

图 19 转盘转速变化图及对应的盘轴位移差变化图

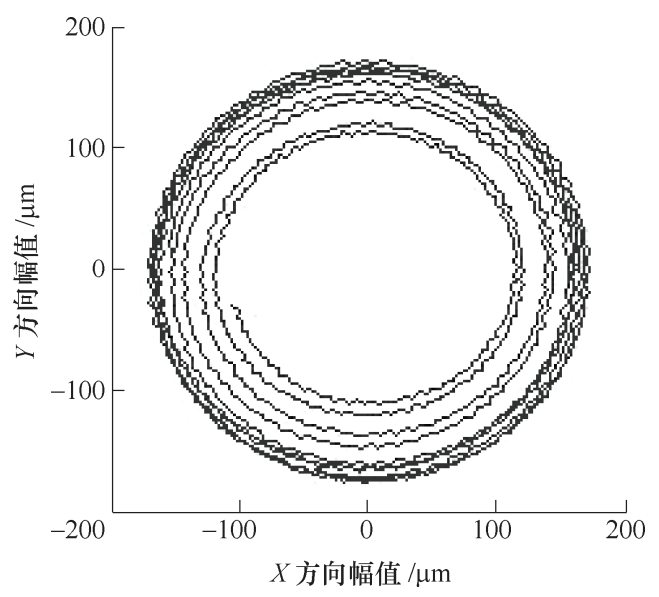

(a) 轴心运动轨迹图

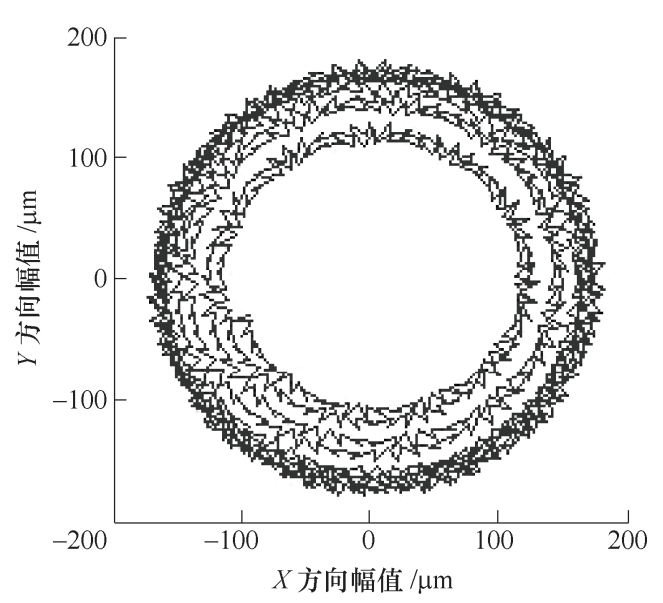

(b) 盘心运动轨迹图

图 20 轴心、盘心运动轨迹图

表 1 运动方程稳态数值结果

\begin{tabular}{|c|c|c|c|c|c|c|c|c|c|}
\hline $\begin{array}{c}\text { 转轴 } x \text { 方向 } \\
\text { 量纲一振 } \\
\text { 幅 } X_{1}\end{array}$ & $\begin{array}{c}\text { 转轴 } x \text { 方向 } \\
\text { 量纲一速 } \\
\text { 度 } X_{1}^{\prime}\end{array}$ & $\begin{array}{c}\text { 转轴 } y \text { 方向 } \\
\text { 量纲一振 } \\
\text { 幅 } Y_{1}\end{array}$ & $\begin{array}{c}\text { 转轴 } y \text { 方向 } \\
\text { 量纲一速 } \\
\text { 度 } Y_{1}^{\prime}\end{array}$ & $\begin{array}{c}\text { 转盘 } x \text { 方向 } \\
\text { 量纲一振 } \\
\text { 幅 } X_{2}\end{array}$ & $\begin{array}{c}\text { 转盘 } x \text { 方向 } \\
\text { 量纲一速度 } \\
X_{2}^{\prime}\end{array}$ & $\begin{array}{c}\text { 转盘 } y \text { 方向 } \\
\text { 量纲一振 } \\
\text { 幅 } Y_{2}\end{array}$ & $\begin{array}{c}\text { 转盘 } y \text { 方向量 } \\
\text { 纲一速度 } Y_{2}^{\prime}\end{array}$ & $\begin{array}{l}\text { 转盘量 } \\
\text { 纲一转 } \\
\text { 速 } \theta^{\prime}\end{array}$ & $\begin{array}{c}\text { 转盘转轴量 } \\
\text { 纲一速度差 } \\
\Delta V\end{array}$ \\
\hline-104.9 & 71.87 & -31.05 & 275.07 & -96.76 & -118.83 & -32.07 & -1236.91 & 0.49 & -1.58 \\
\hline-99.52 & 107.2 & -35.92 & -474.5 & -107.5 & -203.72 & -37.55 & 1016.879 & 0.49 & -1.51 \\
\hline-101.7 & -157.2 & -42.32 & 228.47 & -94.64 & 610.152 & -38.18 & -1086.08 & 0.49 & -2.54 \\
\hline-96.78 & 327.96 & -44.61 & -346.6 & -102.2 & -824.76 & -50.8 & 646.2146 & 0.49 & 0.858 \\
\hline-96.83 & -307.1 & -52.84 & 46.697 & -93.72 & 1100.56 & -45.24 & -525.648 & 0.49 & 1.66 \\
\hline-93.61 & 429.4 & -53.57 & -117.1 & -94.1 & -1089.3 & -61.77 & -25.1887 & 0.49 & -2.04 \\
\hline-90.64 & -312.4 & -62.16 & -193.1 & -92.78 & 1155.31 & -54.24 & 212.8125 & 0.49 & -2.76 \\
\hline-89.53 & 373.7 & -62.91 & 124.82 & -84.94 & -884.25 & -69.71 & -730.002 & 0.49 & 0.81 \\
\hline-83.73 & -166.1 & -70.14 & -389.3 & -90.27 & 753.435 & -65.18 & 824.0948 & 0.49 & -1.76 \\
\hline-84.07 & 187.65 & -72.4 & 277.16 & -76.29 & -290.37 & -74.98 & -1162.49 & 0.49 & 8.053 \\
\hline
\end{tabular}

\section{3 试验研究}

本文设计了盘轴松动故障转子试验台, 以便于 验证仿真结果的正确性, 如图 21 所示, 该实验台由 动力输出系统和盘轴松动故障转子系统组成。其中 假设挠性联轴器为分界点, 左半部分为由转盘、转
轴、轴承座组成的间隙配合盘轴松动故障转子系统; 右半部分为由电机、转轴、挠性联轴节、键相器等 组成的动力输出部分; 挠性联轴器左边和一根 $320 \mathrm{~mm}$ 的转轴相连, 挠性联轴器右边与一根 $500 \mathrm{~mm}$ 的转轴相连, 转轴的两端用滑动轴承支撑, 两轴承座中心之间的跨度 $422 \mathrm{~mm}$ 。试验电机为输 出功率为 $250 \mathrm{~W}$ 的直流电动机, 转轴直径 $9.5 \mathrm{~mm}$, 
转盘外径 $76.2 \mathrm{~mm}$, 转盘质量 $0.612 \mathrm{~kg}$ 。

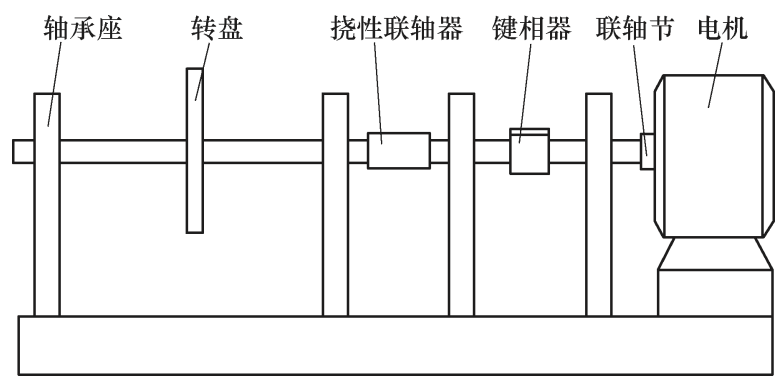

图 21 ZT-3 转子试验台示意图

图 22 为转盘转轴同速时 (转动频率为 $44 \mathrm{~Hz}$ ), $x$ 方向的波形图和频谱图。由图 $22 \mathrm{~b}$ 可知, 频谱图 中既有一倍频, 也有二倍频和三倍频等高阶谐波成 分, 随着高阶谐波的倍频数的增加, 其量纲一幅值 在逐渐减小。对比图 22 与图 16, 可得出实验结果 和仿真结果相似。

图 23 为转盘与转轴转速接近时 (转盘转动频率 为 $34.8 \mathrm{~Hz}$, 转轴转动频率为 $35 \mathrm{~Hz}), x$ 方向波形 图和频谱图。由图 23 可知, 拍振现象也出现了; 其 频谱图中包含一倍频、接近一倍频的低倍频和高倍 频成分, 且在一倍频幅值左右出现多个相邻的频率 幅值。多个相邻频率幅值的出现是由转盘转速不稳 定造成的。对比图 23 和图 17, 两者波形图中均被 发现有拍振现象产生, 两者的结果也是非常类似。 所不同的是, 仿真结果的频谱图中包含频率密集的 高倍频成分, 而实验结果的频谱图中包含多个高倍 频成分。

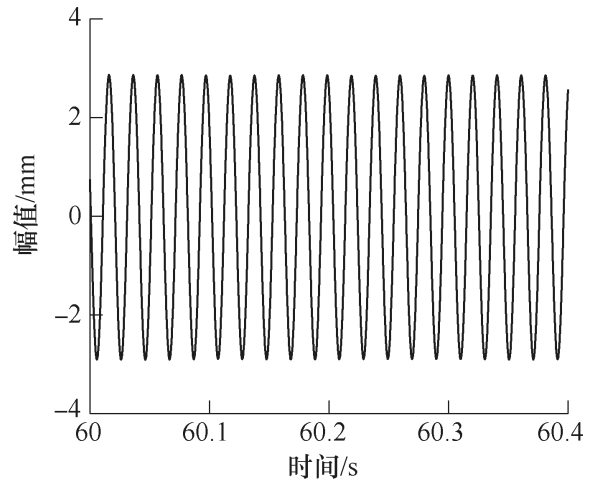

(a) 波形图

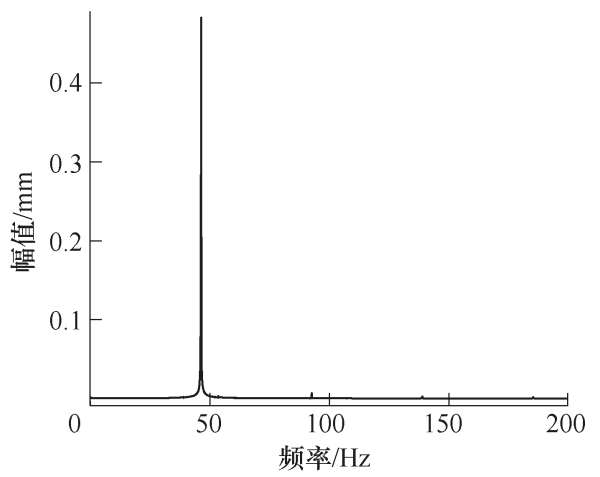

(b) 频谱图

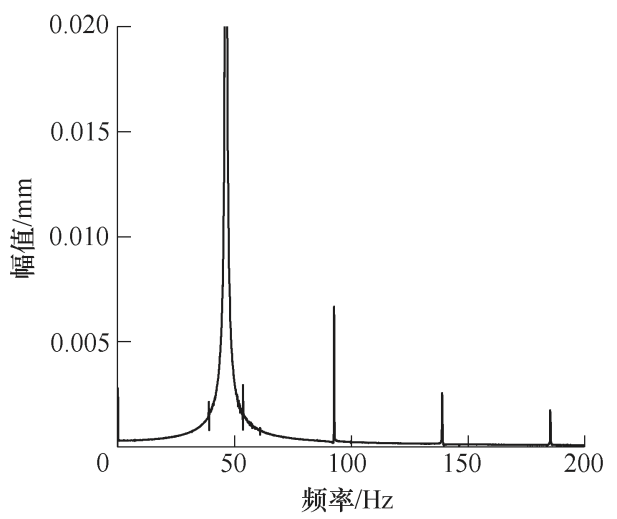

(c) 局部放大图

图 22 波形图和频谱图

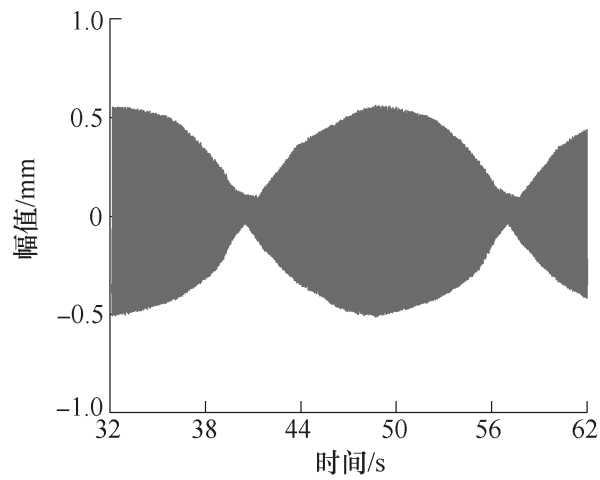

(a) 波形图

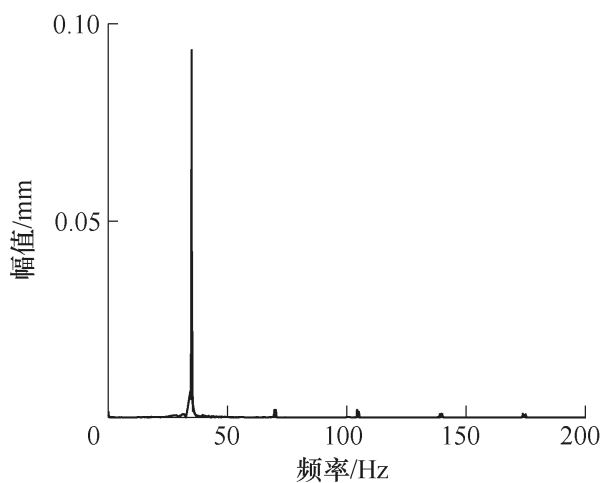

(b) 频谱图

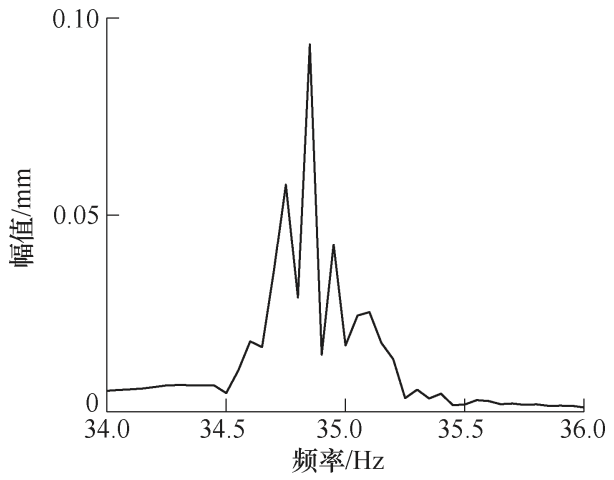

(c) 局部放大图

图 $23 x$ 方向幅频图

图 24 为盘轴转速相差较大时 (转盘转动频率为 $35 \mathrm{~Hz}$, 转轴转动频率为 $17 \mathrm{~Hz}), x$ 方向波形图和 频谱图。由图 24 可知, 拍振现象在波形图中也存在, 
其频谱图中包含转盘转动频率、转轴转动频率和高 倍频频率, 其中高倍频量纲一幅值随着频率增加而 降低。对比图 24 与图 18, 由于仿真结果与实验结 果频谱图中高倍频成分存在一定误差, 因而两者的 波形图也存在一定的差别, 高倍频成分随着盘轴频 率差的增加而增多。

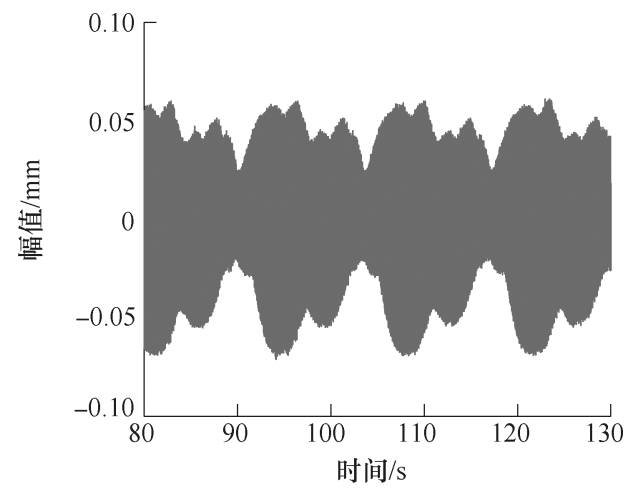

(a) 波形图

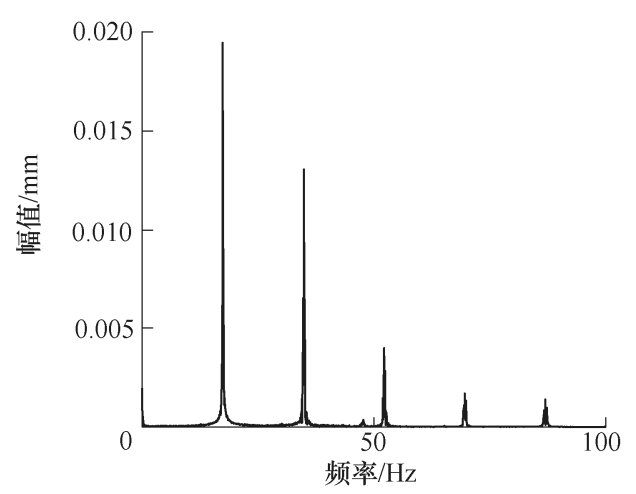

(b) 频谱图

图 24 波形及频谱图

图 25 为转盘的运动轨迹图和局部放大图。由 图 25a 可知, 转盘的运动轨迹是接近于圆形, 由 图 $25 \mathrm{~b}$ 可知, 其运动轨迹存在一定的不规则性, 图 形出现明显的锯齿现象, 锯齿的方向也是不定的, 试验结果总体上和仿真结果基本一致, 从而进一步 验证所建立模型的合理性。

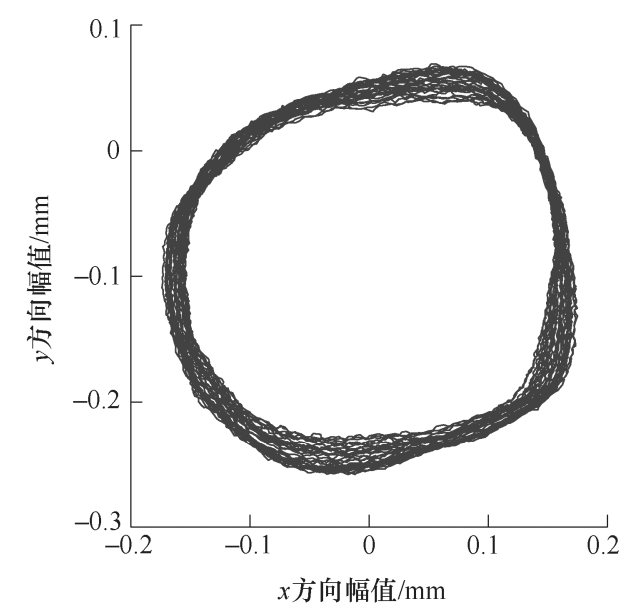

(a) 轨迹图

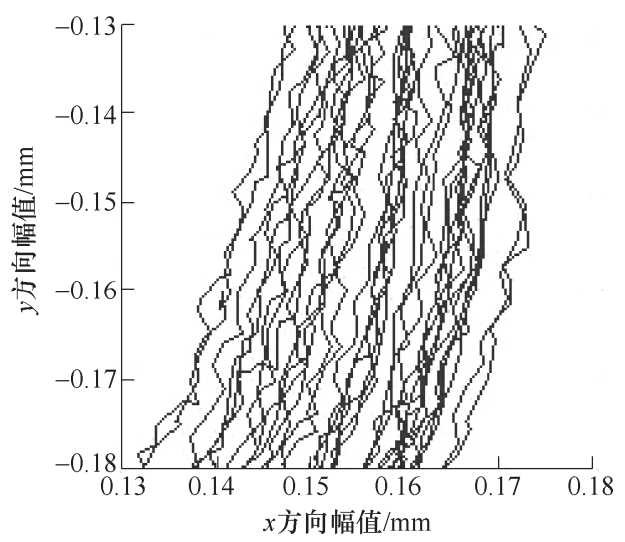

(b) 放大图

图 25 轨迹图和放大图

\section{4 结论}

本文基于 Hertz 碰撞理论建立了间隙配合盘 轴松动转子系统模型, 并根据提出的模型进行了 仿真分析, 最后采用试验结果验证了建立的模型 的合理性。

(1) 转轴转速 $\Omega$ 、盘轴弹性模量 $E_{1} 、 E_{2}$ 、盘 轴间隙 $h$ 、转轴阻尼 $\xi_{1}$ 、转盘阻尼 $\xi_{2}$ 、转动阻尼 $\xi_{3}$ 、转盘初始转速 $\theta_{0}^{\prime}$ 对转盘运动状态都有明显的 影响。

(2) 在一些转速下, 拍振现象会出现在转轴 的波形图中, 这种拍振现象可能是由盘轴碰撞 或盘轴碰摩造成的; 其频谱图中既包含 1 倍频 和接近 1 倍频的成分, 也包含高倍频成分, 且 高倍频的频率成分随着转盘转轴频率差的增加 而增大。

(3) 随转盘转速趋于平稳, 盘轴位移差也趋于 稳定。

(4) 转盘转轴运动轨迹图中均存在锯齿现象, 这种现象是由转盘转轴之间的碰摩造成的; 碰摩力 做功的不同导致锯齿方向的不同，同时也会加剧锯 齿现象。

(5) 转盘与转轴的转速相同时, $x$ 方向的频谱图 中含有二倍频、三倍频, 且随着高次谐波的增加, 其幅值是在不断减小的。当转盘转速接近转轴转速 时, 拍振现象出现在波形图, 频谱图中会出现接近 转盘转动频率和转轴转动频率的频率成分。由于拍 振现象, 在转盘转速附近会出现多个峰值, 频谱图 中也会包含多个高倍频成分。当转盘转速与转轴转 速相差较大时, 拍振现象在波形图中也会类似出现, 高倍频成分也会产生, 且频谱图中的高倍频幅值随 着频率的增加而减小。 


\section{参 考 文 献}

[1] 于欢, 马艳红, 肖森, 等. 高速柔性转子支承松动力学 特征及动力特性 $[\mathrm{J}]$. 北京航空航天大学学报, 2017, 43(8): 1677-1683.

YU Huan, MA Yanhong, XIAO Sen, et al. Mechanical and dynamic characteristics of bearing with looseness on high-speed flexible rotor[J]. Journal of Beijing University of Aeronautics and Astronautics, 2017, 43(8): 1677-1683.

[2] 陈果. 转子一滚动轴承一机匣耦合系统的不平衡/松动 耦合故障非线性动力学 [J]. 机械工程学报, 2008 , 44(3): 82-88.

CHEN Guo. Nonlinear dynamics of unbalance-looseness coupling fults of rotor-ball bearing-stator coupling system[J]. Chinese Journal of Mechanical Engineering, 2008, 44(3): 82-88.

[3] 䒘品先. 有界噪声激励下基座松动/碰摩故障转子动力 学行为的实验研究[D]. 杭州: 浙江大学, 2016 .

RUI Pinxian. experimental research on dynamic behavior of a rotor with base looseness/rub fault under bounded noise excitation [D]. Hangzhou: Zhejiang University, 2016.

[4] 徐洪志, 王南飞, 蒋东翔. 双转子系统轴承座松动的动 力学模型及故障特性 [J]. 航空动力学报, 2016, 31(11): 2781-2794.

XU Hongzhi, WANG Nanfei, JIANG Dongxiang. Bearing pedestal looseness dynamic model of dual rotor system and fault feature [J]. Journal of Aerospace Power, 2016, 31(11): 2781-2794.

[5] 王海飞, 陈果, 廖仲坤, 等. 含支承松动故障的弹用浴 扇发动机整机振动建模与机匣响应特性 $[\mathrm{J}]$. 航空动力 学报, 2015, 30(3): 627-638.

WANG Haifei, CHEN Guo, LIAO Zhongkun, et al. Vibration modeling and casing response characteristics of an integral turbofan engine with support loose fault [J]. Journal of Aerodynamics, 2015, 30(3): 627-638.

[6] 刘杨, 太兴宇, 马辉, 等. 双盘三支撑转子轴承系统松 动-碰摩耦合故障分析 [J]. 航空动力学报, 2013, 28(5): 977-982.

LIU Yang, TAI Xingyu, MA Hui, et al. Loosenessrubbing coupling fault of dual-disk three-support rotorbearing system[J]. Journal of Aerospace Power, 2013, 28(5): 977-982.

[7] 马辉, 太兴宇, 汪博, 等. 松动一碰摩耦合故障转子系 统动力学特性分析 $[\mathrm{J}]$. 机械工程学报, 2012, 48(19):
80-86.

MA Hui, TAI Xingyu, WANG Bo, et al. Dynamic characteristic analysis of a rotor system with pedestal looseness coupled rub-impact fault $[\mathrm{J}]$. Journal of Vibration Engineering, 2012，48(19): 80-86.

[8] 王海飞, 陈果. 连接件松动的非同步振动响应特征分析 与验证 [J]. 工程力学, 2016, 33(4): 225-232.

WANG Haifei, CHEN Guo. Analysis and verification of characteristics of non-synchronous vibration response of loose joints [J]. Engineering Mechanics, 2016, 33(4): 225-232.

[9] GOLDMAN P , MUSZYNSKA A. Analytical and experimental simulation of loose pedestal dynamic effects of a rotating machine vibrational response [J]. Rotating Machinery and Vehicle Dynamics, ASME, 1991, 35: 11-17.

[10] CHU Fulei, TANG Yun. Stability and non-linear response of a rotor-bearing system with pedestal looseness[J]. Journal of Sound and Vibration, 2001, 241(5): 879-893.

[11] LU Wenxiu, CHU Fulei. Experimental investigation of pedestal looseness in a rotor-bearing system[J]. Key Engineering Materials, 2009, 413-414: 599-605.

[12] MUSZYNSKA A, GOLDMAN P. Chaotic responses of unbalanced rotor/bearing/stator systems with looseness or rubs[J]. Chaos Solitons \& Fractals, 1995, 5(9): 1683-1704.

[13] MUSZYNSKA A. Rotordynamics [M]. Boca Raton, FL: CRC Press, 2005.

[14] 张靖, 李鹤, 闻邦椿. 动态油膜-转子系统的支座松动 故障研究 $[J]$. 东北大学学报, 2003, 24(10): 962-965. ZHANG Jing, LI He, WEN Bangchun. On the pedestal looseness of dynamic oil film-rotor system[J]. Journal of Northeastern University, 2003, 24(10): 962-965.

[15] 张靖, 闻邦椿. 带有两端支座松动故障的转子系统的振 动分析[J]. 应用力学学报, 2004, 20(3): 67-71.

ZHANG Jing, WEN Bangchun. Vibration characteristics of a rotor system with pedestal looseness at two supports [J]. Chinese Journal of Applied Mechanics, 2004, 20(3): 67-71.

[16] JIANG Mian, WU Jigang, PENG Xinsheng, et al. Nonlinearity measure based assessment method for pedestal looseness of bearing-rotor systems[J]. Journal of Sound and Vibration, 2017, 411. 
[17] BEHZAD M, ASAYESH M. Vibration analysis of rotating shaft with loose disk, IJE Transactions B: Applications, 2002, 15(4): 385-393.

[18] BEHZAD M, ASAYESH M. Numerical and experimental investigation of the vibration of rotors with loose discs [J]. Mechanical Engineering Science, Proc. IMechE Part C, 2010, 224: 85-94.

[19] 杨绪灿, 金建三. 弹性力学[M]. 北京: 高等教育出版 社, 1987.

YANG Xucan, JIN Jiansan. Elasticity [M]. Beijing: Higher Education Press, 1987.
[20] TIMOSHENKO S, GOODIER J N. Theory of elasticity [M]. McGraw Book Company, Inc., 1951.

作者简介: 李志农, 男, 1966 年出生, 教授, 博士研究生导师。主要研 究方向为转子动力学和机械故障诊断。

E-mail: lizhinong@tsinghua.org.cn

刘杰, 男, 1991 年出生, 硕士研究生。主要研究方向为转子动力学。

E-mail: liujiecome@qq.com

卢文秀(通信作者), 男, 1974 年出生, 副教授, 硕士研究生导师。主要 研究方向为转子动力学。

E-mail: luwenxiu@mail.tsinghua.edu.cn

褚福否, 男, 1959 年出生, 教授, 博士研究生导师。主要研究方向为 转子动力学。

E-mail: chufl@mail.tsinghua.edu.cn

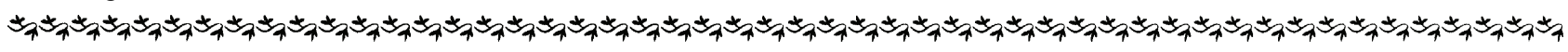

\section{混合磨料柔性抛光工具加工蓝宝石衬底技术研究}

作 者: 许永超

毕业学校: 华侨大学

指导教师: 徐西鹏 陆 静

由于蓝宝石材料硬度大、脆性高和耐腐蚀，传统的铜抛和化学机械抛光方法存在着加工效率低、成本高和污染环境等缺 点。本文基于固相反应原理提出了软硬混合磨料抛光蓝宝石祄底的新构想, 开展了针对蓝宝石祄底的混合磨料抛光技术研究。

通过理论和试验的方法研究了不同种类软磨料与蓝宝石在研抛条件下的反应活性。研究表明在摩擦力诱导的条件下，二 氧化硅较氧化镁和氧化铁磨料对蓝宝石具有更高的反应活性, 最易与蓝宝石发生固相化学反应生成硅酸铝盐。

通过制备混合磨料半固结柔性抛光盘进行蓝宝石祄底的抛光试验。结果表明当软质磨料的化学反应作用和硬质磨料的机 械去除作用达到动态平衡时, 软硬混合磨料较单一硬质磨料的材料去除率提高了 $52.63 \%$, 同时表面粗粘度 Sa 降低了 $19.55 \%$ 。 分析了软硬混合磨料的去除机理，建立了混合磨料的材料去除模型。经过机械接触-反应层预生成-反应层生成-反应层去除四 个阶段实现了材料去除。

探索了高活性磨料和环境友好型的酸碱性加工环境协调混合磨料加工过程中化学作用与机械作用之间的匹配性。结果表 明采用高活性软磨料和合适的有机酸碱化合物有效提高了加工表面质量和加工效率。

通过制备核壳结构的复合磨料改变软磨料的受力方式以促进混合磨料的加工性能。结果表明金刚石/羟基氧化铁复合磨料 能够有效减小晶片表面划痕深度，且羟基氧化铁壳层能够增强抛光盘基体对金刚石磨粒的把持力，有效地延长了抛光工具的 使用寿命。

提出了以混合磨料柔性抛光工具和气液辅助化学机械抛光的新工艺取代传统的铜抛和化学机械抛光工艺来获得超光滑 无损伤表面的新工艺，使得蓝宝石晶片在粗抛光阶段的加工时间缩短至 $50 \mathrm{~min}$ ，加工效率提高了将近 $65 \%$ 。

本文的研究丰富了蓝宝石祄底抛光技术的思路和方法, 对实现蓝宝石祄底的高效率、高质量和无污染的抛光加工具有现 实指导意义。 